\title{
AN INSTABILITY PROPERTY OF THE NONLINEAR SCHRÖDINGER EQUATION ON $S^{d}$
}

\author{
N. Burq, P. Gérard, and N. Tzvetkov
}

\begin{abstract}
We consider the NLS on spheres. We describe the nonlinear evolutions of the highest weight spherical harmonics. As a consequence, in contrast with the flat torus, we obtain that the flow map fails to be uniformly continuous for Sobolev regularity above the threshold suggested by a simple scaling argument.
\end{abstract}

\section{Introduction}

In [4], we obtained a set of Strichartz inequalities as well as local and global well-posedness results for the nonlinear Schrödinger equation (NLS) posed on a compact riemannian manifold $(M, g)$

$$
i \partial_{t} u+\Delta_{g} u=f\left(|u|^{2}\right) u, \quad u(0, x)=u_{0}(x),
$$

where $f$ is a suitably chosen real valued function. A natural question is whether the particular structure of $(M, g)$ influences the critical threshold for the local well-posedness Sobolev regularity of the initial data. Our goal here is to give an affirmative answer to that question. Let us precise what we call local wellposedness in this paper.

Definition 1.1. (see e.g. [9]) We say that the Cauchy problem (1.1) is locally well-posed for data in the Sobolev space $H^{s}(M)$ if for any $R>0$ there exist $T>0$ and a functional space $X_{T}$ continuously embedded in $C\left([-T, T], H^{s}(M)\right)$ and invariant under the natural action of the isometries of $M$, such that for every

$$
u_{0} \in B_{R}:=\left\{u_{0} \in H^{s}(M):\left\|u_{0}\right\|_{H^{s}(M)}<R\right\}
$$

the Cauchy problem (1.1) has a unique solution $u \in X_{T}$. Moreover

1. The map $u_{0} \mapsto u$ is uniformly continuous from $B_{R}$ to $C\left([-T, T], H^{s}(M)\right)$.

2. If $u_{0} \in H^{1}(M), u \in C\left([-T, T], H^{1}(M)\right)$ and satisfies the usual conservation laws

$$
\begin{gathered}
\|u(t)\|_{L^{2}(M)}=\left\|u_{0}\right\|_{L^{2}(M)}, \\
\left\|\nabla_{g} u(t)\right\|_{L^{2}(M)}+\int_{M} F\left(|u(t, x)|^{2}\right) d x=\mathrm{const},
\end{gathered}
$$

where $F$ is a primitive of $f$.

Received November 27, 2001.

2000 Mathematics Subject Classification. 35Q55, 35BXX, 37K05, 37L50, 81Q20.

Key words and phrases. nonlinear Schrödinger, eigenfunctions, dispersive equations. 
The assumption of uniform continuity of the flow map in the above definition seems to be natural for semilinear equations. Moreover it turns out to be the case in the local well-posedness results of [4]. However, as it was pointed to us by $\mathrm{H}$. Koch, such an assumption is unrealistic in the context of quasilinear problems.

The main issue in our analysis is to study the nonlinear evolution by the NLS flow of some eigenfunctions of the Laplace-Beltrami operator on the $d$ dimensional sphere $S^{d}$ with the canonical metric. The situation turns out to be particularly simple in $1 D$. Consider the Cauchy problem

$$
i \partial_{t} u+\partial_{x}^{2} u=|u|^{2} u, \quad u(0, x)=u_{0}(x),
$$

where $x \in S^{1}, t \in \mathbb{R}$. For $s<0$, we set

$$
u_{\kappa, n}(t, x)=\kappa n^{-s} \exp \left(-i t\left(n^{2}+\kappa^{2} n^{-2 s}\right)\right) \exp (i n x) .
$$

It is easy to check that $u_{\kappa, n}$ solves (1.2) with initial data $\kappa n^{-s} \exp (i n x)$. Moreover

$$
\left\|u_{\kappa, n}(t, \cdot)\right\|_{H^{s}\left(S^{1}\right)} \leq|\kappa| .
$$

Fix $\kappa \in] 0,1\left[\right.$. Let $\left\{\kappa_{n}\right\}$ be a sequence of real numbers tending to $\kappa$ which will be specified later. We observe that $u_{\kappa, n}(0, \cdot)-u_{\kappa_{n}, n}(0, \cdot)$ tends to zero in $H^{s}\left(S^{1}\right)$ as $n$ tends to infinity. Take now a positive $t$. Then there exist $C>0$, independent of $n$ and $\delta>0$ such that

$$
\left\|u_{\kappa, n}(t, \cdot)-u_{\kappa_{n}, n}(t, \cdot)\right\|_{H^{s}\left(S^{1}\right)} \geq C\left|\exp \left(-i t n^{-2 s}\left(\kappa^{2}-\kappa_{n}^{2}\right)\right)-1\right|-C n^{-\delta} .
$$

If we suppose that (1.2) is locally well-posed in $H^{s}\left(S^{1}\right), s<0$ then (1.3) would imply

$$
\lim _{n \rightarrow+\infty}\left|\exp \left(-i t n^{-2 s}\left(\kappa^{2}-\kappa_{n}^{2}\right)\right)-1\right|=0
$$

But (1.4) easily fails by choosing $\left\{\kappa_{n}\right\}$ so that

$$
\left(\kappa^{2}-\kappa_{n}^{2}\right) n^{-2 s}=\alpha n^{\beta}
$$

for suitable $\alpha>0, \beta>0$ satisfying $2 s+\beta<0$. This leads to the following result.

Theorem 1. Let $s<0$. Then the Cauchy problem (1.2) is not locally well-posed for data in $H^{s}\left(S^{1}\right)$.

\section{Remark 1.2.}

1. In [2] it is shown that (1.2) is locally well-posed for data in $H^{s}\left(S^{1}\right), s \geq 0$. Therefore the result of Theorem 1 is sharp.

2. The proof of Theorem 1 can be extended to equations of type

$$
i \partial_{t} u+\partial_{x}^{2} u=f\left(|u|^{2}\right) u
$$


under weak assumptions on the nonlinearity, for example $f(\lambda)= \pm \lambda^{\gamma}$, for some $\gamma>0$. In the case of (1.5) one has to deal with the following explicit solution

$$
u_{\kappa, n}(t, x)=\kappa n^{-s} \exp \left(-i t\left(n^{2}+f\left(\kappa^{2} n^{-2 s}\right)\right)\right) \exp (i n x) .
$$

3. It is interesting to mention that if the gauge invariance (if $u$ is a solution of (1.5) then so is $e^{i \theta} u, \theta \in \mathbb{R}$ ) of (1.5) is violated then one can obtain the local well-posedness of the corresponding Cauchy problem for data more singular than $L^{2}\left(S^{1}\right)$. For instance, the Cauchy problem associated to the equation

$$
i \partial_{t} u+\partial_{x}^{2} u=u^{2}
$$

is locally well-posed for data in $H^{s}\left(S^{1}\right), s>-\frac{1}{2}$ (see [8]).

4. A result related to Theorem 1, due to Kenig-Ponce-Vega, when the spatial domain is $\mathbb{R}$ is obtained in [9]. It is shown that the cubic focusing NLS in $1 D$, posed on $\mathbb{R}$, is locally ill-posed for data in $H^{s}(\mathbb{R})$, s $<0$. The construction performed in [9] involves some dilatation arguments. Therefore it seems that the technique of [9] is restricted to the case of data defined on $\mathbb{R}$.

We now turn to the higher dimensional case. Let $(M, g)$ be a two dimensional riemannian manifold and $\Delta_{g}$ be the corresponding Laplace-Beltrami operator. We shall restrict our considerations to cubic defocusing nonlinearity but the arguments can be extended to some other nonlinearities. Thus consider the Cauchy problem

$$
i \partial_{t} u+\Delta_{g} u=|u|^{2} u, \quad u(0, x)=u_{0}(x),
$$

where $x \in M, t \in \mathbb{R}$.

Let $M=\mathbb{R}^{2}$ with the flat metric. Then (1.6) is invariant by a scaling transformation. Namely, if $u(t, x)$ is a solution of (1.6) then so is

$$
u_{\lambda}(t, x)=\lambda u\left(\lambda^{2} t, \lambda x\right)
$$

with initial data $\lambda u_{0}(\lambda x)$. Clearly $\lambda u_{0}(\lambda x)$ has the same $L^{2}\left(\mathbb{R}^{2}\right)$ norm as $u_{0}(x)$. Heuristically this scaling argument suggests that (1.6) is locally well-posed for data in $H^{s}(M), s>0$. Moreover this is the case when $M=\mathbb{R}^{2}$ or $M=\mathbb{T}^{2}$ with the flat metrics, due to [5] in the case $\mathbb{R}^{2}$ and [2] in the periodic case. In this paper we show that the above heuristics fail when $M=S^{2}$.

Theorem 2. Let $T>0, s \in] \frac{3}{20}, \frac{1}{4}[, \kappa \in] 0,1\left[\right.$. Take $M=S^{2}$ with the canonical metric in (1.6). For $n \in \mathbb{N}$, we denote by $\psi_{n}: S^{2} \rightarrow \mathbb{C}$ the restriction to $S^{2}$ of the harmonic polynomial $\left(x_{1}+i x_{2}\right)^{n}$. Then for $t \in[0, T]$ the solution $u_{n}(t)$ of (1.6) with initial data $\kappa \phi_{n}$, where $\phi_{n}=n^{\frac{1}{4}-s} \psi_{n}$ can be represented as

$$
u_{n}(t)=\kappa \exp \left(-i t\left(n(n+1)+\kappa^{2} \omega_{n}\right)\right)\left(\phi_{n}+r_{n}(t)\right),
$$

where $\omega_{n} \approx n^{\frac{1}{2}-2 s}$ and $r_{n}(t)$ satisfies

$$
\left\|r_{n}(t)\right\|_{H^{s}\left(S^{2}\right)} \leq C_{T} n^{-\delta}
$$


where $\delta>0$ and $C_{T}$ depends on $T$ but not on $n$. Moreover there exists $C>0$, independent of $T$ and $n$ such that

$$
\left\|u_{n}\right\|_{L^{\infty}\left(\mathbb{R} ; H^{s}\left(S^{2}\right)\right)} \leq C \kappa .
$$

As a consequence the Cauchy problem (1.6) is not locally well-posed for data in $H^{s}\left(S^{2}\right)$.

\section{Remark 1.3.}

1. The existence of $u \in C^{\infty}\left(\mathbb{R} \times S^{2}\right)$ is guaranteed by Theorem 2 of [4].

2. The condition $s>\frac{3}{20}$ ensures that (1.7) is valid on an arbitrary time interval. If one is interested only in the local well-posedness, a slight modification of the proof of Theorem 2 gives the ill-posedness of the Cauchy problem (1.6) in $H^{s}\left(S^{2}\right), s \in\left[0, \frac{1}{4}\right.$ [ (see section 3.3 below).

3. In [4], we have proved the local well-posedness of (1.6) in $H^{s}(M), s>\frac{1}{2}$. Therefore, in the case $M=S^{2}$ the critical Sobolev regularity for the local well-posedness of (1.6) is in the interval $\left[\frac{1}{4}, \frac{1}{2}\right]$.

4. The choice of $\psi_{n}$ is related to earlier works on spherical harmonics by Stanton-Weinstein [15] and Sogge [14]. In these references, it is proven that $\psi_{n}$ maximizes the quotient $\|\psi\|_{L^{4}} /\|\psi\|_{L^{2}}$ among the spherical harmonics of degree $n$. Moreover, observe that $\psi_{n}$ concentrates on the closed geodesic $x_{1}^{2}+x_{2}^{2}=1$.

The method of proof of Theorem 2 can be further exploited in order to prove ill-posedness results in the energy space for $H^{1}$ subcritical NLS posed on $S^{6}$. These results can be regarded as a consequence of the infinite propagation speed of the Schrödinger operator since similar phenomenon does not occur in the context of $H^{1}$ subcritical semilinear wave equation, with power nonlinearity, posed on $S^{6}$. On the other hand, in the $H^{1}$ supercritical case some semilinear wave equations on $\mathbb{R}^{3}$ are ill-posed in the energy space (see recent works of BrennerKumlin [3] and Lebeau [10]). Counterexamples for subcritical semilinear wave equations containing derivative nonlinearities are constructed in [11].

Theorem 3. Let $\alpha \in] 0,1]$. Then the Cauchy problem

$$
i \partial_{t} u+\Delta_{S^{6}} u=\langle u\rangle^{\alpha} u, \quad u(0, x)=u_{0}(x),
$$

where $x \in S^{6}, t \in \mathbb{R},\langle u\rangle:=\sqrt{1+|u|^{2}}$ is not locally well-posed for data in $H^{1}\left(S^{6}\right)$.

Remark 1.4. The question of extending our results to more general geometries than the sphere is still open. However, if $(M, g)$ is a compact orientable $d$ dimensional riemannian manifold with a closed stable (elliptic) geodesic, then by considering quasimodes for initial data as constructed in [13] (see also [1], [7], [6]), one easily shows that for $s<\frac{d-1}{4}, d \geq 2$ the Picard iteration scheme applied to the integral formulation of the nonlinear Schrödinger equation

$$
i \partial_{t} u+\Delta_{g} u= \pm|u|^{2} u
$$


sends at the second iteration any ball of $H^{s}(M)$ into an unbounded set (see [12] and the references therein for related results in other contexts).

The rest of this note is organized as follows. In Section 2, we collect some algebraic facts which are useful in the sequel. In Section 3, we first give the proof of Theorem 2. Then we extend the argument providing the ill-posedness for $s \in\left[0, \frac{1}{4}\right.$ [. Since the proof of Theorem 3 is very similar to that of Theorem 2 , in section 4 we only give the main lines of the proof of Theorem 3. Finally, at the end of Section 4, we discuss instability features of some weak solutions of (1.10).

\section{Some algebraic lemmas}

In this section, $n$ is a fixed positive integer, and $d \geq 2$. For every $\alpha \in \mathbb{R}$, we denote by $R_{\alpha}$ the rotation of $\mathbb{R}^{d+1}$ defined by

$$
R_{\alpha}\left(x_{1}, x_{2}, x_{3}, . ., x_{d+1}\right)=\left(\cos \alpha x_{1}-\sin \alpha x_{2}, \sin \alpha x_{1}+\cos \alpha x_{2}, x_{3}, . ., x_{d+1}\right)
$$

and by $R_{\alpha}^{\star}$ the associated unitary operator of $L^{2}\left(S^{d}\right)$,

$$
R_{\alpha}^{\star} u(x)=u\left(R_{\alpha}(x)\right) .
$$

For $x \in S^{d}$, we set $\psi(x)=\left(x_{1}+i x_{2}\right)^{n}$. Observe that $R_{\alpha}^{\star} \psi=\exp ($ in $\alpha) \psi$ for every $\alpha \in \mathbb{R}$.

Lemma 2.1. Let $u \in L^{2}\left(S^{d}\right)$ satisfying, for every $\alpha \in \mathbb{R}$

$$
R_{\alpha}^{\star} u=\exp (\text { in } \alpha) u .
$$

Then the decomposition of $u$ into spherical harmonics reads

$$
u=\omega \psi+\sum_{j} g_{j}
$$

where $\omega \in \mathbb{C}$ and each $g_{j}$ is a spherical harmonic of degree $>n$.

2.1. Proof of Lemma 2.1. Since the family $\left(R_{\alpha}^{\star}\right)_{\alpha \in \mathbb{R}}$ is a one-parameter group of unitary operators leaving invariant the space of spherical harmonics of degree $l$, one can find an orthonormal basis $\left(h_{k}\right)$ of $L^{2}\left(S^{d}\right)$ such that, for every $k, h_{k}$ is a spherical harmonic satisfying, for some $n_{k} \in \mathbb{Z}$, for every $\alpha \in \mathbb{R}$,

$$
R_{\alpha}^{\star} h_{k}=\exp \left(i n_{k} \alpha\right) h_{k} .
$$

Comparing (2.1) and (2.2), the decomposition of $u$ in the basis $\left(h_{k}\right)$ reads

$$
u=\sum_{k: n_{k}=n} c_{k} h_{k}
$$

Let $h$ be a spherical harmonic of degree $l$ satisfying property (2.1) for every $\alpha \in \mathbb{R}$. Denote by $P$ the $l$-homogeneous polynomial on $\mathbb{R}^{d+1}$ such that $h=P_{\mid S^{d}}$. Then (2.1) is equivalent to

$$
\forall x \in \mathbb{R}^{d+1}, \quad P\left(R_{\alpha}(x)\right)=\exp (\text { in } \alpha) P(x) .
$$


Let us decompose $P$ according to the powers of $z=x_{1}+i x_{2}$ and $\bar{z}$

$$
P\left(x_{1}, x_{2}, x_{3}, . ., x_{d+1}\right)=\sum_{p+q \leq l} z^{p} \bar{z}^{q} A_{p q}\left(x_{3}, . ., x_{d+1}\right)
$$

where $A_{p q}$ is a homogeneous polynomial of degree $l-p-q$. In view of $(2.4)$, (2.5) and

$$
P\left(R_{\alpha}(x)\right)=\sum_{p+q \leq l} e^{i(p-q) \alpha} z^{p} \bar{z}^{q} A_{p q}\left(x_{3}, . ., x_{d+1}\right),
$$

we conclude that $A_{p q}=0$ unless $p-q=n$. As a consequence,

$$
l \geq p+q \geq p-q=n
$$

and, if $l=n$, then $p=n$ and $q=0$, so that $P=c z^{n}$, i.e. $h=c \psi$. Coming back to decomposition (2.3), this completes the proof.

The main advantage of property (2.1) is that it is invariant under the nonlinear transformations

$$
u \longmapsto f\left(|u|^{2}\right) u \text {. }
$$

Combining this remark with Lemma 2.1, we obtain the following two statements.

Lemma 2.2. Let $f \in L_{l o c}^{\infty}\left(\mathbb{R}_{+}\right)$. Then there exists $\omega \in \mathbb{C}$ such that

$$
f\left(|\psi|^{2}\right) \psi=\omega \psi+r
$$

where $r$ is a sum of spherical harmonics of degree $>n$.

Lemma 2.3. Let $c \in \mathbb{C}$ and $f \in C^{\infty}\left(\mathbb{R}_{+}\right)$, such that (1.1) is locally well-posed (see Definition 1.1) in $H^{s}(M)$. Let $u \in C\left([-T, T], H^{s}(M)\right) \cap X_{T}$ be the solution of (1.1) satisfying $u(0)=c \psi$. Then for every $t \in[-T, T]$,

$$
u(t)=\omega(t) \psi+\sum_{j} g_{j}(t),
$$

where $\omega(t) \in \mathbb{C}$ and each $g_{j}(t)$ is a spherical harmonic of degree $>n$.

Lemma 2.2 is an immediate consequence of Lemma 2.1 and the above remark. Lemma 2.3 follows from Lemma 2.1 and the observation that $R_{\alpha}^{\star} u$ and $\exp ($ in $\alpha) u$ belong to $C\left([-T, T], H^{s}(M)\right) \cap X_{T}$ and solve (1.1) with the same Cauchy data $c \exp ($ in $\alpha) \psi$.

\section{Proof of Theorem 2}

We drop the subscript $n$ for conciseness. We first prove (1.9). Recall the following conservation laws for (1.6).

$$
\begin{aligned}
\|u(t)\|_{L^{2}}^{2} & =\kappa^{2}\|\phi\|_{L^{2}}^{2}, \\
\|\nabla u(t)\|_{L^{2}}^{2}+\frac{1}{2}\|u(t)\|_{L^{4}}^{4} & =\kappa^{2}\|\nabla \phi\|_{L^{2}}^{2}+\frac{\kappa^{4}}{2}\|\phi\|_{L^{4}}^{4} .
\end{aligned}
$$

The identity (3.1) results from the gauge invariance of (1.6) while (3.2) is due to the time translation invariance of (1.6). Since one has explicit asymptotics with 
respect to $n$ of all Sobolev and Lebesgue norms of $\phi$ one can obtain bounds for $u$. First a use of (3.1) yields

$$
\|u\|_{L^{\infty}\left(\mathbb{R} ; L^{2}\right)} \leq C \kappa n^{-s} .
$$

Next using (3.2), we infer that

$$
\begin{aligned}
\|\nabla u\|_{L^{\infty}\left(\mathbb{R} ; L^{2}\right)} & \leq C \kappa\left(\|\nabla \phi\|_{L^{2}}+\|\phi\|_{L^{4}}^{2}\right) \\
& \leq C \kappa n^{1-s} .
\end{aligned}
$$

An interpolation argument gives

$$
\begin{aligned}
\|u\|_{L^{\infty}\left(\mathbb{R} ; H^{s}\right)} & \leq\|u\|_{L^{\infty}\left(\mathbb{R} ; L^{2}\right)}^{1-s}\|u\|_{L^{\infty}\left(\mathbb{R} ; H^{1}\right)}^{s} \\
& \leq C \kappa
\end{aligned}
$$

which proves (1.9).

Further we set $c(t):=\exp \left(-i t\left(n(n+1)+\kappa^{2} \omega\right)\right)$, where

$$
\omega:=\frac{\|\phi\|_{L^{4}}^{4}}{\|\phi\|_{L^{2}}^{2}} \approx n^{\frac{1}{2}-2 s} .
$$

Then $c(t)$ satisfies the equation

$$
i c_{t}-n(n+1) c=\kappa^{2} \omega|c|^{2} c .
$$

Write $u(t)=\kappa c(t)(\phi+w(t))$. Using Lemma 2.2 and (3.3), we obtain that the equation for $w(t)$ is

$$
i w_{t}+\left(\Delta_{S^{2}}+n(n+1)+\kappa^{2} \omega\right) w=\kappa^{2}\left(|\phi+w|^{2}(\phi+w)-|\phi|^{2} \phi+r\right) .
$$

with $w(0)=0$ and

$$
r \in \overline{\oplus_{l \geq n+1} \operatorname{Ker}\left(\Delta_{S^{2}}+l(l+1)\right)} .
$$

Further we decompose $w(t)=z(t) \phi+q(t)$ for some $z: \mathbb{R} \rightarrow \mathbb{C}$ and $q: \mathbb{R} \rightarrow$ $C^{\infty}\left(S^{2}\right)$. Then due to Lemma 2.3, we can suppose

$$
q(t) \in \overline{\oplus_{l \geq n+1} \operatorname{Ker}\left(\Delta_{S^{2}}+l(l+1)\right)}
$$

The proof of Theorem 2 will be completed ${ }^{1}$ once we prove the following two lemmas.

Lemma 3.1. There exists a constant $C>0$, independent of $T$ and $n$ such that

$$
\|q(t)\|_{H^{s}} \leq C n^{-\frac{1}{4}-s} .
$$

Lemma 3.2. There exists a constant $C_{T}>0$, which depends on $T$ but not on $n$ such that

$$
\sup _{t \in[0, T]}|z(t)| \leq C_{T} n^{\frac{1}{4}-3 s} .
$$

\footnotetext{
${ }^{1}$ The ill-posedness in $H^{s}\left(S^{2}\right)$ follows from an argument similar to the one in $1 D$ already performed in the introduction.
} 
3.1. Proof of Lemma 3.1. We first rewrite the conservation laws (3.1), (3.2) in terms of $z(t)$ and $q(t)$. Since $\phi$ is orthogonal to $q(t)$ in $L^{2}\left(S^{2}\right)$ as well as $\nabla \phi$ to $\nabla q(t)$, we can rewrite (3.1) and (3.2) as

$$
|1+z(t)|^{2}\|\phi\|_{L^{2}}^{2}+\|q(t)\|_{L^{2}}^{2}=\|\phi\|_{L^{2}}^{2},
$$

Observe that

$$
\|\nabla \phi\|_{L^{2}}^{2}=\left(\phi \mid-\Delta_{S^{2}} \phi\right)=n(n+1)\|\phi\|_{L^{2}}^{2},
$$

where $(\cdot \mid \cdot)$ denotes the $L^{2}\left(S^{2}\right)$ scalar product. Therefore multiplying (3.6) with $-n(n+1)$ and adding it to $(3.7)$ gives

$$
\begin{aligned}
\|\nabla q(t)\|_{L^{2}}^{2}-n(n+1)\|q(t)\|_{L^{2}}^{2} & \leq \frac{\kappa^{2}}{2}\|\phi\|_{L^{4}}^{4} \\
& \leq C n^{\frac{1}{2}-4 s} .
\end{aligned}
$$

Due to (3.5), we can write $q(t)=\sum_{l \geq n+1} q_{l}(t)$ where $q_{l} \in \operatorname{Ker}\left(\Delta_{S^{2}}+l(l+1)\right)$. Hence

$$
\left(3.8\|\nabla q(t)\|_{L^{2}}^{2}-n(n+1)\|q(t)\|_{L^{2}}^{2}=\sum_{l \geq n+1}(l(l+1)-n(n+1))\left\|q_{l}(t)\right\|_{L^{2}}^{2} .\right.
$$

If $l \geq n+1$, the following inequalities hold

$$
l(l+1)-n(n+1) \geq C n
$$

and

$$
\frac{l(l+1)-n(n+1)}{l(l+1)} \geq C n^{-1} .
$$

Therefore we arrive at

$$
\|q(t)\|_{L^{2}}^{2} \leq C n^{-\frac{1}{2}-4 s}
$$

and

$$
\|\nabla q(t)\|_{L^{2}}^{2} \leq C n^{\frac{3}{2}-4 s} .
$$

An interpolation argument yields

$$
\begin{aligned}
\|q(t)\|_{H^{s}} & \leq\|q(t)\|_{L^{2}}^{1-s}\|q(t)\|_{H^{1}}^{s} \\
& \leq C n^{-\frac{1}{4}-s} .
\end{aligned}
$$

This completes the proof of Lemma 3.1. 
3.2. Proof of Lemma 3.2. We project the equation (3.4) on $\phi$ and obtain the following equation for $z(t)$

$$
\begin{aligned}
i z_{t}+\omega \kappa^{2} z= & \frac{\kappa^{2}}{\|\phi\|_{L^{2}}^{2}}\left(\left(|\phi+w|^{2}(\phi+w) \mid \phi\right)-\left(|\phi|^{2} \phi \mid \phi\right)\right) \\
= & \frac{\kappa^{2}}{\|\phi\|_{L^{2}}^{2}}\left\{\int\left(2|\phi|^{2} w+\phi^{2} \bar{w}\right) \bar{\phi}\right. \\
& \left.+\int\left(2 \operatorname{Re}(\bar{\phi} w) w \bar{\phi}+|w|^{2}|\phi|^{2}+|w|^{2} w \bar{\phi}\right)\right\} .
\end{aligned}
$$

Recall that $w(t)=z(t) \phi+q(t)$ and $|\phi|^{2} \phi=\omega \phi+r$. Therefore the equation for $z(t)$ can be rewritten as

$$
\begin{aligned}
i z_{t}+\omega \kappa^{2} z=2 \omega \kappa^{2} z+\omega \kappa^{2} \bar{z}+ & \frac{\kappa^{2}}{\|\phi\|_{L^{2}}^{2}} \mathcal{O}\left(|z|^{2} \int|\phi|^{4}+|z|^{3} \int|\phi|^{4}\right. \\
& \left.+\int|q|^{3}|\phi|+\int|q|^{2}|\phi|^{2}+|(q \mid r)|\right) .
\end{aligned}
$$

We first estimate the source terms. Write

$$
\begin{aligned}
\frac{\int|q|^{3}|\phi|}{\|\phi\|_{L^{2}}^{2}} & \leq C n^{2 s}\|q\|_{L^{3}}^{3}\|\phi\|_{L^{\infty}} \\
& \leq C n^{2 s}\|q\|_{L^{2}}^{2}\|\nabla q\|_{L^{2}}\|\phi\|_{L^{\infty}} \\
& \leq C n^{2 s} n^{-\frac{1}{2}-4 s} n^{\frac{3}{4}-2 s} n^{\frac{1}{4}-s} \\
& =C n^{\frac{1}{2}-5 s} .
\end{aligned}
$$

Further we have

$$
\begin{aligned}
\frac{\int|q|^{2}|\phi|^{2}}{\|\phi\|_{L^{2}}^{2}} & \leq C n^{2 s}\|q\|_{L^{2}}^{2}\|\phi\|_{L^{\infty}}^{2} \\
& \leq C n^{2 s} n^{-\frac{1}{2}-4 s} n^{\frac{1}{2}-2 s} \\
& =C n^{-4 s}
\end{aligned}
$$

and

$$
\begin{aligned}
\frac{|(q \mid r)|}{\|\phi\|_{L^{2}}^{2}} & \leq C n^{2 s}\|q\|_{L^{2}}\|r\|_{L^{2}} \\
& \leq C n^{2 s} n^{-\frac{1}{4}-2 s} n^{\frac{1}{2}-3 s} \\
& =C n^{\frac{1}{4}-3 s} .
\end{aligned}
$$

Therefore if $s>\frac{1}{8}$ the equation for $z(t)$ can be written as

$$
i z_{t}=2 \omega \kappa^{2} \operatorname{Re}(z)+\mathcal{O}\left(\omega|z|^{2}+\omega|z|^{3}+n^{\frac{1}{4}-3 s}\right)
$$

with $z(0)=0$. Moreover using once again the $L^{2}$ conservation law (3.6), we have

$$
1-|1+z|^{2}=\frac{\|q(t)\|_{L^{2}}^{2}}{\|\phi\|_{L^{2}}^{2}}=\mathcal{O}\left(n^{-\frac{1}{2}-2 s}\right) .
$$


Therefore

$$
\left.|2 \operatorname{Re}(z)+| z\right|^{2} \mid=\mathcal{O}\left(n^{-\frac{1}{2}-2 s}\right)
$$

and the equation (3.9) takes the form

$$
i z_{t}=\mathcal{O}\left(\omega|z|^{2}+\omega|z|^{3}+n^{\frac{1}{4}-3 s}\right),
$$

with $\omega=\mathcal{O}\left(n^{\frac{1}{2}-2 s}\right)$. Hence if we set

$$
M(T)=\sup _{0 \leq t \leq T}|z(t)|,
$$

we obtain

$$
M(T) \leq C T\left(n^{\frac{1}{2}-2 s}\left([M(T)]^{2}+[M(T)]^{3}\right)+n^{\frac{1}{4}-3 s}\right) .
$$

In view of (3.10), we set

$$
\tilde{M}(T)=n^{3 s-\frac{1}{4}} M(T)
$$

and therefore (3.10) yields

$$
\tilde{M}(T) \leq C T\left(1+n^{\frac{3}{4}-5 s}\left([\tilde{M}(T)]^{2}+n^{\frac{1}{4}-3 s}[\tilde{M}(T)]^{3}\right)\right) .
$$

Since $\tilde{M}(0)=0$ and $s>\frac{3}{20}$, we obtain that $\tilde{M} \leq C T$ uniformly with respect to $n$. This completes the proof of Lemma 3.2.

3.3. Ill-posedness for $s \in\left[0, \frac{3}{20}\right]$. In order to prove that (1.6) is not locally well-posed for data in $H^{s}\left(S^{2}\right), s \in\left[0, \frac{3}{20}\right]$ one needs to slightly extend the argument of section 3.2. Suppose that (1.6) is locally well-posed for data in $H^{s}\left(S^{2}\right)$, $s \in\left[0, \frac{3}{20}\right]$. Using the above method, we need to show only that (1.7) holds on a small time interval $\left[0, T_{n}\right]$ such that

$$
\lim _{n \mapsto+\infty} n^{\frac{1}{2}-2 s} T_{n}=+\infty
$$

since the argument providing the ill-posedness performed in the introduction works equally well provided that (3.11) holds. Therefore, with the notation of the previous section, one needs a bound for $M(T)$ only for $T \in\left[0, T_{n}\right]$ with $T_{n}$ satisfying (3.11). One now defines $\tilde{M}(T)$ as

$$
\tilde{M}(T)=T_{n}^{-1} n^{3 s-\frac{1}{4}} M(T)
$$

and therefore for $T \in\left[0, T_{n}\right]$ one has

$$
\tilde{M}(T) \leq C\left(1+T_{n}^{2} n^{\frac{3}{4}-5 s}\left([\tilde{M}(T)]^{2}+T_{n} n^{\frac{1}{4}-3 s}[\tilde{M}(T)]^{3}\right)\right) .
$$

Let $\varepsilon>0$ be a small number to be fixed later. For $s \geq 0$, we choose $T_{n}$ as follows

$$
T_{n}:=n^{\frac{5}{2} s-\frac{3}{8}-\varepsilon} \text {. }
$$

Clearly with the above choice of $T_{n}$ the relation (3.11) holds provided $\varepsilon \ll 1$. Moreover, we have a uniform bound for $\tilde{M}(T), T \in\left[0, T_{n}\right]$. This implies that for $t \in\left[0, T_{n}\right]$ one has $|z(t)| \leq c n^{-\frac{1}{2} s-\frac{1}{8}-\varepsilon}$ which proves the validity of (1.7) the interval $\left[0, T_{n}\right]$. 


\section{Sketch of the proof of Theorem 3}

Suppose that (1.10) is locally well-posed in $H^{1}\left(S^{6}\right)$. Let $\psi: S^{6} \rightarrow \mathbb{C}$ be the restriction to $S^{6}$ of the harmonic polynomial $\left(x_{1}+i x_{2}\right)^{n}$. Then it is easy to see that $\|\psi\|_{L^{q}} \approx n^{-\frac{5}{2 q}}$. Consider (1.10) with Cauchy data $u(0)=\kappa \phi:=\kappa n^{\frac{1}{4}} \psi$, where $\kappa \in\left[\frac{1}{2}, 1\right]$. Then $\|\phi\|_{H^{1}} \approx 1$ and due to Lemma 2.1 there exists $\omega \in \mathbb{C}$ and $r$ so that

$$
\left(\kappa^{-2}+|\phi|^{2}\right)^{\alpha / 2} \phi=\omega \phi+r,
$$

where $r$ is a sum of spherical harmonics of degree $>n$. Moreover, we can check that uniformly in $\kappa \in\left[\frac{1}{2}, 1\right]$,

$$
\omega=c^{\star} n^{\alpha / 4}+\mathcal{O}\left(n^{-\delta}\right),
$$

where $\delta>0$ and $c^{\star}$ is independent of $\kappa$. Now we set $c(t):=\exp (-i t(n(n+$ $\left.5)+\kappa^{\alpha} \omega\right)$ ). Let $u(t)$ be the solution of (1.10) subject to initial data $\phi$. Writing $u(t)=\kappa c(t)(\phi+w(t))$ we obtain that the equation for $w(t)$ is

$$
\begin{aligned}
i w_{t}+\left(\Delta_{S^{6}}+n(n+5)+\kappa^{\alpha} \omega\right) w & \\
& =\kappa^{\alpha}\left(\left(\kappa^{-2}+|\phi+w|^{2}\right)^{\alpha / 2}(\phi+w)-\left(\kappa^{-2}+|\phi|^{2}\right)^{\alpha / 2} \phi+r\right) .
\end{aligned}
$$

Let $w(t)=z(t) \phi+q(t)$. We now give the estimates of the quantities involved in the argument, performed in details in the previous section.

Estimate for $\|r\|_{L^{2}}$. Clearly

$$
\|r\|_{L^{2}} \leq C\left\|\phi^{\alpha+1}\right\|_{L^{2}} \leq C n^{\frac{\alpha-4}{4}} .
$$

Estimate for $\|q(t)\|_{L^{2}}$. Similarly to the previous section one can check that

$$
n\|q(t)\|_{L^{2}}^{2} \leq C\|\phi\|_{L^{\alpha+2}}^{\alpha+2}
$$

and therefore

$$
\|q(t)\|_{L^{2}} \leq C n^{\frac{\alpha-12}{8}} \text {. }
$$

Therefore, using the above bounds for $\|r\|_{L^{2}}$ and $\|q(t)\|_{L^{2}}$, we obtain

$$
\frac{|(q(t) \mid r)|}{\|\phi\|_{L^{2}}^{2}} \leq C n^{\frac{3 \alpha-4}{8}} \text {. }
$$

and

$$
\frac{\|q(t)\|_{L^{2}}^{2}}{\|\phi\|_{L^{2}}^{2}} \leq C n^{\frac{\alpha-4}{4}}
$$

Estimate for $\|q(t)\|_{H^{1}}$. Clearly

$$
\|q(t)\|_{H^{1}} \leq C n^{1+\frac{\alpha-12}{8}} \leq C n^{-\delta},
$$

where $\delta>0$ provided $\alpha<4$.

Analysis on the nonlinear term. Set

$$
F_{\phi}(w, \bar{w})=\left(\kappa^{-2}+|\phi+w|^{2}\right)^{\alpha / 2}(\phi+w)-\left(\kappa^{-2}+|\phi|^{2}\right)^{\alpha / 2} \phi .
$$


Then $F_{\phi}(0,0)=0$ and

$$
\begin{aligned}
\frac{\partial F_{\phi}}{\partial w}(0,0) & =\left(\kappa^{-2}+|\phi|^{2}\right)^{\alpha / 2}+\frac{\alpha}{2}|\phi|^{2}\left(\kappa^{-2}+|\phi|^{2}\right)^{\alpha / 2-1}, \\
\frac{\partial F_{\phi}}{\partial \bar{w}}(0,0) & =\frac{\alpha}{2} \phi^{2}\left(\kappa^{-2}+|\phi|^{2}\right)^{\alpha / 2-1} .
\end{aligned}
$$

Since $\alpha \leq 1$, the second derivatives of $F_{\phi}$ are bounded and therefore, similarly to the previous section, the equation for $z(t)$ can be written as

$$
i z_{t}=\mathcal{O}\left(n^{\frac{\alpha}{4}}|z|^{2}+n^{\frac{3 \alpha-4}{8}}\right)+\frac{1}{\|\phi\|_{L^{2}}^{2}} \mathcal{O}\left(\int|z \phi+q|^{2}|\phi|\right) .
$$

Estimate for $\int|z \phi+q|^{2}|\phi|$. Write

$$
\begin{aligned}
\frac{\int|z \phi+q|^{2}|\phi|}{\|\phi\|_{L^{2}}^{2}} & \leq \frac{C}{\|\phi\|_{L^{2}}^{2}}\left(|z|^{2} \int|\phi|^{3}+\|q\|_{L^{2}}^{2}\|\phi\|_{L^{\infty}}\right) \\
& \leq \mathcal{O}\left(n^{\frac{1}{4}}|z|^{2}\right)+\mathcal{O}\left(n^{\frac{\alpha-3}{4}}\right) .
\end{aligned}
$$

Since $\alpha \in] 0,1]$ the equation for $z$ becomes

$$
i z_{t}=\mathcal{O}\left(n^{\frac{1}{4}}|z|^{2}+n^{\frac{3 \alpha-4}{8}}\right)
$$

with $z(0)=0$. Now we set

$$
M(T):=\sup _{0 \leq t \leq T}|z(t)|
$$

We look for a $T_{n}$ such that

$$
\lim _{n \mapsto+\infty} n^{\frac{\alpha}{4}} T_{n}=+\infty
$$

with a bound for $M(T), T \in\left[0, T_{n}\right]$. Now we set

$$
\tilde{M}(T)=T_{n}^{-1} n^{\frac{4-3 \alpha}{8}} M(T)
$$

and therefore for $T \in\left[0, T_{n}\right]$ one has

$$
\tilde{M}(T) \leq C\left(1+T_{n}^{2} n^{\frac{3 \alpha-2}{8}}[\tilde{M}(T)]^{2}\right) .
$$

Since $\alpha$ is positive we can take $T_{n}$ so that $n^{-\frac{\alpha}{4}} \ll T_{n} \ll n^{\frac{2-3 \alpha}{16}}$ and therefore the representation $u(t)=\kappa c(t) \phi+\mathcal{O}\left(n^{-\gamma}\right)$ in $H^{s}$ holds for $t \in\left[0, T_{n}\right]$ for some $\gamma>0$. Now it remains to perform the argument of the introduction in order to prove the ill-posedness.

Remark 4.1. Global weak solutions of (1.10) in the class $H^{1}$ can be obtained by a standard compactness argument. The method consists of considering a regularized version of (1.10), involving a small parameter. The Cauchy problem associated to the regularized equation possesses unique global solution, due to control on high Sobolev norms. Then one passes into the limit as the small parameter tends to zero. This is possible due to the $H^{1}$ bound on the solutions of (1.10). Note that if the regularized version of (1.10) is gauge invariant then one may apply Lemma 2.3 to the regularized equation and therefore there exists a global weak solution of (1.10) with data $\phi$ (see the previous section for the 
definition of $\phi)$ such that its spectral decomposition at any fixed time $t$ contains only spherical harmonics of degree $\geq n$. Due to the argument of the previous section we obtain that this solution has strongly instability features when letting $n$ tend to infinity.

\section{Acknowledgement}

We thank H. Koch for discussions on the notion of local well-posedness.

\section{References}

[1] V.M. Babich and V.F. Lazutkin, Eigenfunction concentrated near a closed geodesic, Topics in Math. Phys. Vol. 2 (M. S. Birman, ed.), Consultant's Bureau, New York, 1968, 9-18.

[2] J. Bourgain, Fourier transform restriction phenomena for certain lattice subsets and application to nonlinear evolution equations I. Schrödinger equations, Geom. Funct. Anal. 3 (1993), no. 2, 107-156.

[3] P. Brenner and P. Kumlin, On wave equations with supercritical nonlinearities, Arch. Math. (Basel) 74 (2000), no. 2, 129-147.

[4] N. Burq, P. Gérard and N. Tzvetkov, Strichartz inequalities and the nonlinear Schrödinger equation on compact manifolds, preprint, 2001.

[5] T. Cazenave and F. Weissler, The Cauchy problem for the critical nonlinear Schrödinger equation in $H^{s}$, Nonlinear Anal. 14 (1990), no. 10, 807-836.

[6] Y. Colin de Verdière, Quasi-modes sur les variétés Riemanniennes, Invent. Math. 43 (1977), no. 1, 15-52.

[7] V. Guillemin and A. Weinstein. Eigenvalues associated with a closed geodesic, Bull. Amer. Math. Soc. 82 (1976), no. 1, 92-94.

[8] C. Kenig, G. Ponce and L. Vega, Quadratic forms for the $1-D$ semilinear Schrödinger equation, Trans. Amer. Math. Soc. 348 (1996), no. 8, 3323-3353.

[9] , On the ill-posedness of some canonical dispersive equations, Duke Math. J. 106 (2001), no. 3, 617-633.

[10] G. Lebeau, Non linear optic and supercritical wave equation, preprint 2001. (see also Optique non linéaire et ondes sur critiques, Séminaire EDP, 1999-2000, Exp. No. IV, 13 pp., Sémin. Équ. Dériv. Partielles, Ecole Polytech., Palaiseau, 2000.)

[11] H. Lindblad, A sharp counterexample to the local existence of low-regularity solutions to nonlinear wave equations, Duke Math. J. 72 (1993), no. 2, 503-539.

[12] L. Molinet, J. C. Saut and N. Tzvetkov, Well-posedness and ill-posedness results for the Kadomtsev-Petviashvili-I equation, Duke Math. J., to appear.

[13] J. Ralston, Approximate eigenfunctions of the Laplacian, J. Differential Geom. 12 (1977), no. $1,87-100$.

[14] C. Sogge, Oscillatory integrals and spherical harmonics, Duke Math. J. 53 (1986), no. 1, $43-65$.

[15] R. Stanton and A. Weinstein, On the $L^{4}$ norm of spherical harmonics, Math. Proc. Cambridge Philos. Soc. 89 (1981), no. 2, 343-358.

Université Paris Sud, Mathématiques, BÂt 425, 91405 Orsay Cedex, France.

E-mail address: Nicolas.burq@math.u-psud.fr

E-mail address: Patrick.gerard@math.u-psud.fr

E-mail address: Nikolay.tzvetkov@math.u-psud.fr 